\title{
Asymptomatic peripheral nerve dysfunction and vascular reactivity in IDDM patients with and without microalbuminuria
}

\author{
C.W. Bodmer, E. A. Masson, M.W. Savage, S. Benbow, A.W. Patrick, G. Williams \\ Department of Medicine, University of Liverpool, Liverpool, UK
}

Summary Abnormal vascular reactivity has been implicated in the aetiology of diabetic microvascular disease and we have previously demonstrated enhanced contractility of hand veins to noradrenaline in insulin-dependent diabetic (IDDM) patients with microalbuminuria. We have now assessed the possible contribution of subclinical peripheral nerve dysfunction to exaggerated vascular reactivity in microalbuminuric patients. Twenty-five IDDM patients (15 with microalbuminuria), none of whom had symptomatic neuropathy, and 10 control subjects were studied. Vasoconstrictor responses were measured in dorsal hand veins using noradrenaline and phenylephrine. Conduction in median, peroneal and sural nerves was assessed using electrophysiology, and autonomic function using standard cardiovascular reflex tests. The noradrenaline dose causing $50 \%$ vasoconstriction was significantly lower in the microalbuminuric diabetic subjects compared with normoalbuminuric (3.6(1.7) mean (SEM) $\mathrm{ng} / \mathrm{min}$ vs 20.1(6.0) $\mathrm{ng} / \mathrm{min}, p=0.0002)$ and non-diabetic subjects (35.1(5.0) ng/min; $p<0.0001)$. However, reactivity to phenylephrine did not differ between the groups. Median nerve motor conduction velocity was significantly slower in microalbuminuric (48.4(1.4) $\mathrm{m} / \mathrm{s}$ ) than in normoalbuminuric $(52.7(1.2) \mathrm{m} / \mathrm{s}$, $p=0.04)$ and non-diabetic subjects $(56.7(0.9) \mathrm{m} / \mathrm{s}$, $p=0.0001)$. In the diabetic group overall, there was a strongly positive linear correlation between vascular response to noradrenaline and conduction velocity in both the median nerve $(r=0.62, p=0.0009)$ and peroneal nerve $(r=0.53, p=0.006)$. There was no correlation between phenylephrine-induced responses and motor conduction velocity in either nerve, nor were indices of autonomic function correlated with vascular reactivity to either agent. Enhanced vascular reactivity to noradrenaline $\left(\alpha_{1}\right.$ - and $\alpha_{2}$-agonist), but not phenylephrine ( $\alpha_{1}$-agonist only), in subclinical neuropathy is correlated with severity of nerve damage. This suggests that damage to presynaptic inhibitory $\alpha_{2}$-adrenoceptors is in part responsible for the exaggerated vascular responses found in microalbuminuric subjects. [Diabetologia (1994) 37: 1056-1061]

Key words Vascular reactivity, peripheral neuropathy, $\alpha$-adrenoceptors, nerve conduction, microalbuminuria.
Received: 23 February 1994

and in revised form: 8 June 1994

Corresponding author: Dr. C.W. Bodmer, Diabetes Centre, Ipswich Hospital, Heath Road, Ipswich IP4 5PD, UK

Abbreviations: NA, Noradrenaline; PE, phenylephrine; MCV, motor conduction velocity; $\mathrm{ED}_{50}$, dose of drug estimated to cause $50 \%$ vasoconstriction.
Vascular tone is determined by neural and hormonal influences, including noradrenaline (NA) [1], which acts on vascular smooth muscle $\alpha$-adrenoceptors to cause vasoconstriction. These receptors can be stimulated by NA released locally from sympathetic nerve endings, and into the circulation from nerve endings and the adrenal medulla. We have previously reported that vascular reactivity to NA is exaggerated in normotensive insulin-dependent diabetic (IDDM) patients with incipient nephropathy [2]. We measured vascular responses in veins on the dorsum of 
Table 1. Characteristics of subjects studied

\begin{tabular}{llll}
\hline & IDDM Patients & Non-diabetic & $\begin{array}{l}\text { Nonbjects } \\
\text { subjects }\end{array}$ \\
\cline { 2 - 3 } & Microalbuminuria & Normoalbuminuria & 10 \\
Age (years) & 15 & 10 & $34.2(3.8)$ \\
Duration of diabetes (years) & $40.7(3.7)$ & $43.4(2.5)^{\mathrm{a}}$ & - \\
MAP (mm Hg) & $20.3(2.3)$ & $22.2(3.1)$ & $84.1(1.8)$ \\
HbA $(\%)$ & $99.6(1.9)^{\mathrm{a}}$ & $98.3(3.8)^{\mathrm{a}}$ & - \\
AER (mg/24 h) & $11.2(0.6)$ & $10.3(0.5)$ & - \\
\hline
\end{tabular}

Data are presented as mean (SEM). ${ }^{a} p<0.01$ vs non-diabetic control subjects; ${ }^{b} p<0.0001$ vs subjects with normal albumin excretion.

the hand, which carry $\alpha$-adrenoceptors and other vascular receptors $[3,4]$, and which therefore have been used as a model of vascular responses in resistance vessels. This exaggerated response could cause an increase in systemic blood pressure via increased peripheral resistance and, within the kidney, might lead to raised intraglomerular pressure, one of the factors which predisposes to albuminuria. A hyperresponsive vasculature could, therefore, have a role in the aetiology and progression of microvascular disease in diabetes.

NA acts on both pre- (vasodilator) and post-synaptic (vasoconstrictor) $\alpha_{2}$-adrenoceptors, as well as post-synaptic (vasoconstrictor) $\alpha_{1}$-adrenoceptors. It is not clear whether the abnormally increased responsiveness to NA in microalbuminuric patients is due to an intrinsic defect of the $\alpha$-adrenoceptor, the post-receptor pathways or an increase in receptor numbers. Another possible factor is damage to sympathetic nerve endings. This could impair release of NA, resulting in 'up-regulation' of post-synaptic (vasoconstrictor) receptors and so to 'denervation hypersensitivity', with an exaggerated response to exogenous NA. Alternatively, loss of the pre-synaptic $\alpha_{2}$-adrenoceptors, which mediate reuptake of NA into the nerve terminal and inhibit NA release from the sympathetic ending, could explain enhanced vascular responsiveness to NA.

We therefore attempted to clarify the cause of increased NA responsiveness in two ways. Firstly, to identify the receptor subtype responsible, we compared responses to phenylephrine (PE), which acts specifically on post-synaptic $\alpha_{1}$-adrenoceptors and has no pre-synaptic effect [5]. An abnormally increased response to $\mathrm{PE}$ as well as NA would suggest an intrinsic abnormality of $\alpha_{1}$-adrenoceptors, or their post-receptor pathways, whereas a normal response to PE would point to a defect of $\alpha_{2}$-adrenoceptors. Secondly, to determine the role of peripheral nerve function, we have studied the relationship between vascular responses and sensitive indices of nerve function. In our original study [2], subjects had no clinical features of sensorimotor or autonomic neuropathy, but we did not seek evidence of subclinical
MAP, Mean arterial pressure, calculated from the equation: $1 / 3$ (systolic-diastolic) + diastolic. AER, albumin excretion rate (median of three collections)

nerve damage, which is common in patients with IDDM of many years' duration. Although there is individual variation, all fibre types tend to be affected in diabetic peripheral neuropathy [6], and tests of small fibre function, for example thermal sensory thresholds [7], are less reliable and reproducible than large-fibre conduction velocity, which is the best index of large-fibre integrity [8]. We therefore examined peripheral nerve function using nerve conduction velocity and vascular reactivity to NA and $\mathrm{PE}$ in dorsal hand veins.

\section{Subjects and methods}

Subjects. Twenty-five IDDM patients and 10 non-diabetic subjects were studied (Table 1). All subjects were normotensive by World Health Organisation criteria [9] and the diabetic patients had no symptoms or signs of peripheral or autonomic neuropathy. Patients with electrophysiological evidence of nerve entrapment were excluded. None of the patients had any clinical evidence of macrovascular disease. Fourteen patients had microalbuminuria (urinary albumin $30-300 \mathrm{mg} / 24 \mathrm{~h}$ in two out of three urine collections), but none had overt diabetic nephropathy (proteinuria greater than $0.5 \mathrm{~g} / 24 \mathrm{~h}$ ). All subjects were non-smokers, free of other illness and were taking no medication other than insulin. Subjects were instructed to avoid caffeine-containing drinks and alcohol for $12 \mathrm{~h}$ before study.

This study was approved by the Ethics Committee of the Royal Liverpool University Hospital, and fully-informed written consent was obtained from each subject.

Vascular response studies. Vasoconstrictor responses to NA and $\mathrm{PE}$ were measured in a vein on the dorsum of the hand. The subjects lay supine in a temperature-controlled (20$22^{\circ} \mathrm{C}$ ) room with one arm resting at $30^{\circ}$ above the horizontal. The vein under study in the elevated arm was cannulated and a sphygmomanometer cuff attached around the upper arm.

Changes in vein diameter were measured using a linear variable differential transducer (LVDT; model $100 \mathrm{MHR}$, Schaevitz Engineering, Pennsauken, N.J., USA) and an ATA 101 signal conditioner (Schaevitz Engineering). The LVDT was mounted on the back of the hand using a tripod with flexible feet. The tip of the LVDT's core was positioned over the centre of the vein approximately $1 \mathrm{~cm}$ downstream (proximal) from the tip of the needle.

After a 30-min run-in period, baseline measurements of vein diameter were taken with the sphygmomanometer cuff 
Table 2. Infusion rates estimated to cause $50 \%$ vasoconstriction $\left(\mathrm{ED}_{50}\right)$ to noradrenaline (NA) and phenylephrine (PE), and motor conduction velocity (MCV)

\begin{tabular}{llll}
\hline & \multicolumn{2}{l}{ IDDM patients } & Non-diabetic subjects \\
\cline { 2 - 3 } & Microalbuminuria & Normoalbuminuria & \\
\hline$n$ & $15^{\mathrm{a}}$ & $10^{\mathrm{a}}$ & 10 \\
$\mathrm{ED}_{50} \mathrm{NA}(\mathrm{ng} / \mathrm{min})$ & $3.6(1.7)^{\mathrm{b}}$ & $20.1(6.0)$ & $35.1(5.0)$ \\
$\mathrm{ED}_{50} \mathrm{PE}(\mu \mathrm{g} / \mathrm{min})$ & $0.78(0.64)$ & $1.88(1.45)$ & $0.81(0.32)$ \\
$\mathrm{MCV}\left(\mathrm{m} / \mathrm{s}^{\mathrm{c}}\right.$ & & & \\
Median nerve & $48.4(1.4)^{\mathrm{d}}$ & $52.7(1.2)^{\mathrm{e}}$ & $56.7(0.9)$ \\
Peroneal nerve & $40.6(1.8)^{\mathrm{d}}$ & $46.1(2.1)$ & $49.8(2.2)$ \\
\hline
\end{tabular}

Data are presented as mean (SEM). ${ }^{a}$ Eight microalbuminuric and seven normoalbuminuric subjects were studied with PE. ${ }^{b} p<0.001$ vs normoalbuminuric diabetic and non-diabetic control subjects; 'Values are for all subjects; for subgroups subjects

first deflated, and then inflated to $45 \mathrm{~mm} \mathrm{Hg}$. The difference between the two readings indicated the maximal vein diameter. The diameter of the vein was measured during infusion of stepped doses of NA (1-32 ng/min, in 5\% dextrose) and expressed as a percentage of the maximal baseline value. Each dose was infused for $5 \mathrm{~min}$ before inflation of the cuff and for 2 min thereafter; vein diameter was measured at the end of this period. All the control subjects and 14 of the diabetic subjects had further hand-vein response studies using PE (1$10,000 \mathrm{ng} / \mathrm{min}$, in $0.9 \% \mathrm{NaCl}$ ). The coefficient of variation for this technique using NA is $9 \%$ [2]. The doses of NA and PE used had no influence on pulse or blood pressure, and the rates of $5 \%$ dextrose infused $(0.1$ or $0.2 \mathrm{ml} / \mathrm{min}$ ) had no influence on plasma glucose concentations.

Nerve conduction studies. Peripheral nerve function was measured using conventional electrophysiological methods with surface electrodes and a Medelec MS6 Electromyograph (Medelec Limited, Old Woking, Surrey, UK) [10]. Recordings were made in the temperature-controlled room $\left(20-22^{\circ} \mathrm{C}\right)$, and skin temperature was maintained at greater than $30^{\circ} \mathrm{C}$ throughout. Motor nerve conduction velocities (MCV) were recorded in the median and peroneal nerves, and sensory nerve conduction velocity and evoked potential amplitude were recorded in the median and sural nerves, ipsilateral to the vascular response studies. Computer averaging was employed for the sensory studies.

Autonomic function tests. Cardiovascular autonomic reflexes were evaluated using established methods [11]. Blood pressure was measured with the patient supine using an automated recorder (Omega 1400, Invivo Research Laboratories, Broken Arrow, O.K., USA) after a 10-min rest, and then after standing for $1 \mathrm{~min}$, to determine the fall in systolic pressure (normal $<10 \mathrm{~mm} \mathrm{Hg}$ ). To assess the heart-rate response to deep breathing, subjects breathed in and out as deeply as possible during $10-\mathrm{s}$ cycles ( $5 \mathrm{~s}$ inspiration, $5 \mathrm{~s}$ expiration) for $1 \mathrm{~min}$. Results are expressed as the maximum difference in R-R interval during this period (normal $>15$ beats $/ \mathrm{min}$ ). The immediate heart rate response to standing was measured after a separate period of 10 min rest, and $\mathrm{R}$ waves counted from the moment of standing. Results are expressed as the ratio of the R-R inter$\mathrm{val}$ at the 30th heart beat after standing, compared with that at the 15 th heart beat (30:15 ratio; normal $>1.04)$. Patients with abnormal autonomic function test results were excluded from the study.

Assays. Urinary albumin concentration was measured by immunoturbidimetry using a kit method (Kone Corporation, studied with PE see text. ${ }^{\mathrm{d}} p<0.05$ vs normoalbuminuric diabetic and non-diabetic control subjects; ${ }^{\mathrm{e}} p<0.05$ vs non-diabetic control subjects

Espoo, Finland), and albumin excretion rate was expressed as the median value from three consecutive 24-h urine collections. The intra- and inter-assay coefficients of variation for this assay were both less than $3 \%$. Glycaemic control was assessed by glycated haemoglobin $\left(\mathrm{HbA}_{1}\right)$ concentration, measured by electroendosmosis (normal range $<8.0 \%$ ).

\section{Statistical analysis}

The results were analysed using the Arcus Pro-II statistical package (Medical Computing, Aughton, West Lancashire, UK). The drug dose estimated to cause $50 \%$ vasoconstriction $\left(\mathrm{ED}_{50}\right)$ was calculated from individual dose-response curves using Probit analysis [12]. Unpaired $t$-tests were used to assess differences between the groups. Pearson's Linear Correlation was used to analyse the relationship between nerve conduction parameters and $\mathrm{ED}_{50}$. $\mathrm{ED}_{50}$ values were not normally distributed and were therefore analysed after log-transformation, which normalised the distribution. Data are presented as mean (SEM).

\section{Results}

Table 1 shows the characteristics of the IDDM diabetic and non-diabetic subjects studied. The control subjects were significantly younger $(p<0.02)$ than the normoalbuminuric diabetic group but not the microalbuminuric group, and there was no difference in age between the two diabetic groups; age has previously been shown not to affect hand-vein reactivity [13]. Mean arterial blood pressure was higher in both diabetic groups in comparison to the control subjects $(p<0.01)$, but there was no difference between the two diabetic groups $(p=0.8)$.

Table 2 shows the $E_{50}$ values for NA- and PE-induced vasoconstriction, and the nerve conduction velocities for all groups. The microalbuminuric group had significantly greater sensitivity (i.e. a lower $\left.\mathrm{ED}_{50}\right)$ to NA than both the normoalbuminuric patients $(p=0.0002)$ and the control $(p<0.0001)$ group; there was no difference in $\mathrm{ED}_{50}$ to NA between these latter two groups $(p=0.1)$. By contrast, there was no difference in PE responsiveness be- 


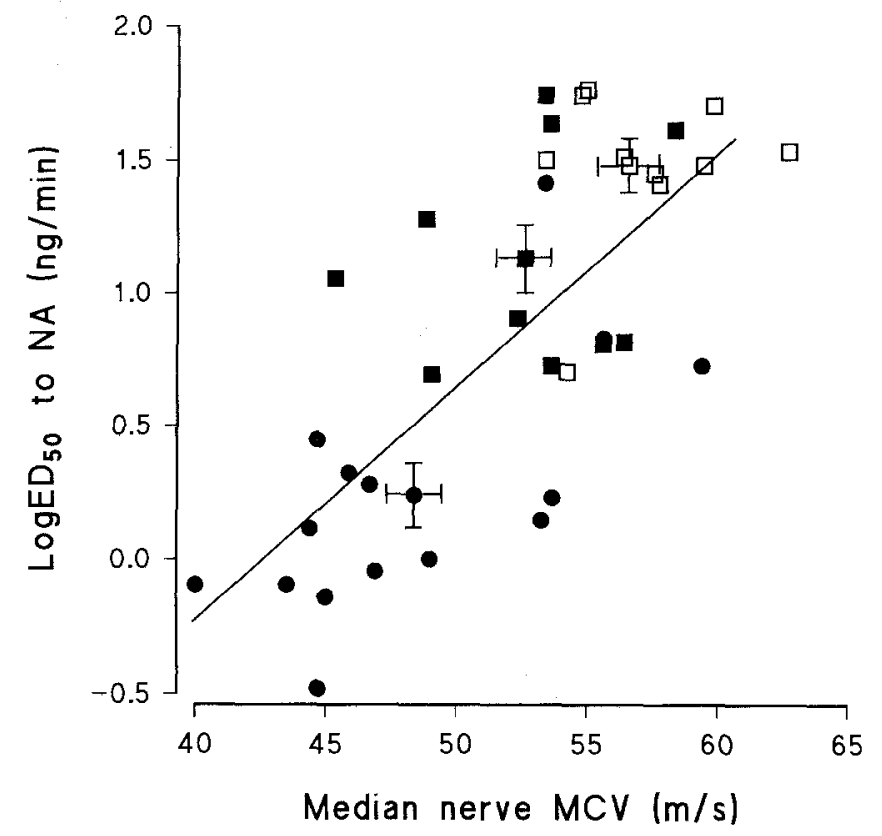

Fig. 1. Correlation between $\log E \mathrm{D}_{50}$ to noradrenaline (NA) and median nerve motor conduction velocity (MCV) in IDDM patients with (•) and without $(\square)$ microalbuminuria, and non-diabetic subjects $(\square) ; r=0.72, p<0.0001$. The regression line for the group as a whole is shown. A lower $\mathrm{ED}_{50}$ indicates greater sensitivity. Error bars indicate mean (SEM) for $\log \mathrm{ED}_{50}$ and median nerve MCV for the three groups

tween any of the three groups $(p>0.4)$. Median nerve MCV was significantly lower in the microalbuminuric group when compared with both the normoalbuminuric $(p=0.04)$ and non-diabetic groups $(p=$ 0.0001 ), and the normoalbuminuric group had a lower MCV than the non-diabetic group $(p=0.01)$. Peroneal nerve $\mathrm{MCV}$ was also significantly lower in the microalbuminuric group than in the other two groups $(p<0.05)$, but there was no difference between the latter.

There was a strong positive linear correlation between $\log \mathrm{ED}_{50}$ to NA and median nerve MCV when all the groups were analysed together $(r=0.72$, $r^{2}=0.52, p<0.0001$; Fig. 1$)$. This correlation was maintained for the diabetic group as a whole $\left(r=0.62, r^{2}=0.38, p=0.0009\right)$ and for the microalbuminuric group $\left(r=0.65, r^{2}=0.43, p=0.008\right)$, but not for the normoalbuminuric group $(r=0.2, p=0.6)$. There was also a positive correlation between $\log \mathrm{ED}_{50}$ for NA and peroneal nerve MCV for all groups $\left(r=0.52, r^{2}=0.27, p=0.001\right)$, but this was not maintained when the groups were analysed separately. There were no significant correlations between $\log \mathrm{ED}_{50}$ for $\mathrm{PE}$ and either median nerve MCV $(50.8(1.6) \mathrm{m} / \mathrm{s} ; r=0.17, p=0.5)$ or peroneal nerve MCV (42.1(1.9) $\mathrm{m} / \mathrm{s} ; r=0.45, p=0.1$ ), when data from all subjects were combined.

There were no significant correlations between vascular reactivity and any of the other nerve func- tion parameters measured. Specifically, $\mathrm{ED}_{50}$ to NA was not significantly correlated with median nerve sensory amplitude $(r=-0.15, p=0.49)$ or velocity $(r=0.24, p=0.26)$, or sural nerve amplitude $(r=0.004, p=0.99)$ or conduction velocity $(r=0.17$, $p=0.41)$. Neither were there significant correlations with standard tests of autonomic function when all subjects were analysed together: 30:15 ratio $(r=0.30, p=0.26)$, systolic fall in blood pressure on standing $(r=0.23, p=0.35)$, change in heart rate during deep breathing $(r=0.20, p=0.45)$, nor when microalbuminuric and normoalbuminuric diabetic, and non-diabetic control subjects were analysed separately. Group analysis showed no relation between blood pressure and $\mathrm{ED}_{50}$ to either NA or PE.

Multiple linear regression analysis showed that median nerve MCV was the only significant predictor of $\mathrm{ED}_{50}$ to NA ( $\left.p=0.0006\right)$, amongst several variables; namely $\mathrm{HbA}_{1}$, systolic, diastolic and mean arterial blood pressure, age, duration of diabetes, and peroneal nerve MCV. As previously reported [2], albumin excretion rates showed an 'either/or' effect; individual values of $\mathrm{AER}$ did not correlate with $\mathrm{ED}_{50}$ or $\mathrm{MCV}$, despite the significant differences described above when subjects were grouped according to whether or not they had microalbuminuria.

\section{Discussion}

In this study, we set out to determine firstly, which subtype of $\alpha$-adrenoceptor mediates the previously described increase in vascular reactivity to NA in microalbuminuric IDDM diabetic subjects [2]; and secondly, to discover whether subclinical nerve damage could contribute. We therefore studied vascular responses to noradrenaline ( $\alpha_{1}-$ and $\alpha_{2}$-agonist) and phenylephrine (pure $\alpha_{1}$-agonist), and their relationship with various measures of nerve function.

This study confirmed the increased NA-induced vasoreactivity found previously in microalbuminuric IDDM subjects. However, there were no differences in response to phenylephrine between the groups. NA acts at both $\alpha_{1}$ - (post-synaptic) and $\alpha_{2}$ - (pre- and post-synaptic) adrenoceptors. $\mathrm{PE}$ is a pure $\alpha_{1}$-adrenoceptor agonist, stimulating these receptors post-synaptically on smooth muscle. Stimulation of post-synaptic $\alpha_{2}$-adrenoceptors results in vasoconstriction, whereas pre-synaptic $\alpha_{2}$-adrenoceptors, on the sympathetic nerve ending, are responsible for the re-uptake of NA from the synaptic cleft and for preventing NA release from the nerve terminal; pre-synaptic $\alpha_{2}$-adrenoceptors are, therefore, inhibitors of vasoconstriction. The findings in our previous study therefore suggested either an increase in post-synaptic or decrease in pre-synaptic $\alpha_{2}$-adrenoceptor activity.

We have also demonstrated a close relationship between MCV velocity and vascular reactivity to 
NA in IDDM subjects without clinically significant neuropathy, although, intriguingly, this correlation was not maintained for sensory conduction velocity. The maintenance of this correlation for the peroneal nerve in the ipsilateral leg indicates that diffuse damage was present throughout the peripheral nervous system, as has been suggested previously [14]. The non-diabetic subjects had significantly better nerve function than both diabetic groups, confirming that nerve fibre damage is widespread in people with long-standing diabetes, even in the absence of clinical evidence of neuropathy. The correlation between vascular reactivity and motor nerve conduction was no longer present when vascular responses were measured with PE. This implies that the differences we have demonstrated are due to changes at the $\alpha_{2}$-adrenoceptor. These findings are in keeping with previous work [15-17], including a recent study [17] which assessed vascular responses using a similar technique. This latter study compared hand vein reactivity with postural drop in blood pressure in IDDM patients with symptomatic autonomic neuropathy, and found a positive correlation with responses to NA but not to $\mathrm{PE}$.

If denervation hypersensitivity were the mechanism responsible for exaggerated vascular responsiveness in neuropathy, then one would expect both post-synaptic $\alpha_{1}$ - and $\alpha_{2}$-adrenoceptors to be upregulated. This is clearly not the case, since we demonstrated no increased response with PE (pure $\alpha_{1}$-adrenoceptor agonist). Therefore, the differences in response to NA between the diabetic and non-diabetic subjects in our study, as well as the correlation between $\mathrm{ED}_{50}$ and $\mathrm{MCV}$, are most likely due to changes in the pre-synaptic $\alpha_{2}$-adrenoceptor, presumably as a result of nerve fibre damage.

Standard tests of central autonomic function in our asymptomatic patients did not correlate with vascular sensitivity to NA or PE. However, sensitive measures of peripheral nerve function demonstrated a close correlation with vascular responses to NA in IDDM patients. We suggest that this could indicate subclinical damage to sympathetic nerve endings. Several tests of peripheral autonomic function have been described, for example sweat testing [18] and galvanic skin responses [19]; these are semiquantitative and have variable degrees of reproducibility. Cardiovascular reflex tests may not be sensitive to minor degrees of autonomic damage in the extremities. Since vascular tone is normally under autonomic nervous control [1], it is possible that peripheral venous responses may be a more sensitive indicator of peripheral autonomic function than either conventional electrophysiology or central cardiovascular reflex tests.

Blood pressure was higher in both diabetic groups in comparison with non-diabetic control subjects, whereas vascular responses to PE were not different between any of these three groups. In addition, re- sponses to NA were different between the two diabetic groups, with the non-diabetic subjects reacting similarly to the normoalbuminuric diabetic patients. Thus, differences in blood pressure cannot account for the altered vascular responses we have found in this study.

In our previous study of vascular reactivity in IDDM patients [2], we found an exaggerated vasoconstrictor response to NA in patients with microalbuminuria, and postulated that this effect could contribute to increased urinary albumin losses, via increased efferent glomerular arteriolar tone and increased intraglomerular pressure, and possibly also to hypertension, via increased peripheral resistance, although blood pressure did not correlate with vascular reactivity in this or our previous study. The difference in vascular reactivity was confirmed in the present study, and was accompanied by reduced median nerve MCV when compared with both normoalbuminuric and non-diabetic groups. Circulating catecholamine levels are reportedly normal or high in patients with microvascular disease [20,21], and we have previously demonstrated no difference in circulating NA levels in diabetic subjects with and without microalbuminuria [2]. Therefore, although there is clearly an association between peripheral nerve dysfunction and vascular reactivity to NA (with approximately $50 \%$ interdetermination, $r^{2}=0.43$ for the microalbuminuric group), it is unlikely that this alone can be responsible for the exaggerated vascular reactivity found in our previous study. It may be that the microalbuminuric IDDM patients also have other factors, such as genetic predisposition [13, 22], which are responsible for their exaggerated responses.

In conclusion, the present study confirmed the exaggerated vascular responsiveness in diabetic subjects with microalbuminuria which we have demonstrated previously. Moreover, we have found a close relationship between vascular reactivity to NA, but not to $\mathrm{PE}$, and peripheral nerve function, even in patients who have no clinical evidence of nerve damage. This implies that, although other factors are also involved, damage to pre-synaptic inhibitory $\alpha_{2}$-adrenoceptors has a role in the exaggerated vasoconstrictor responses found in microalbuminuric IDDM patients.

Acknowledgements. This study was supported by grants from Bayer UK Ltd and Pfizer Ltd, and by charitable donations from the Liverpool Diabetes Research Action Fund. We thank Drs. B.A. Walker and M.C. White for permission to study their patients.

\section{References}

1. Benjamin N, Vallance P (1991) Local control of human peripheral vascular tone: implications for drug therapy. Clin Sci 80:183-190 
2. Bodmer CW, Patrick AW, How TV, Williams G (1992) Exaggerated sensitivity to NE-induced vasoconstriction in IDDM patients with microalbuminuria: possible etiology and diagnostic implications. Diabetes 41:209-214

3. Bulbring E, Tomita T (1987) Catecholamine action on smooth muscle. Pharmacol Rev 39:49-95

4. Vanhoutte PM, Shepherd JT (1985) Adrenergic pharmacology of human and canine peripheral veins. Fed Proc 44:337-340

5. Weiner N (1985) Norepinephrine, epinephrine and the sympathomimetic amines. In: Goodman LS, Gilman A (eds) The pharmacological basis of therapeutics. 7th edn MacMillan, New York, pp 170-171

6. Britland ST, Young RJ, Sharma AK, Clarke BF (1990) Association of painful and painless diabetic polyneuropathy with different patterns of nerve fiber degeneration and regeneration. Diabetes 39:898-908

7. Bodmer CW, Masson EA, Lake D, Benbow SJ, Savage MW, Williams G (1993) Convenience and reproducibility of the Medoc 2001 thermal sensory analyser. Diabet Med 10 [Suppl 1]:S20-S21

8. Veves A, Malik RA, Lye RH et al. (1991) The relationship between sural nerve morphometric findings and measures of peripheral nerve function in mild diabetic neuropathy. Diabet Med 8:917-921

9. Memorandum from the WHO/ISH (1986) 1986 guidelines for the treatment of mild hypertension. Hypertension 8:957-961

10. Malik RA, Masson EA, Sharma AK et al. (1990) Hypoxic neuropathy: relevance to human diabetic neuropathy. Diabetologia 33:311-318

11. Ewing DJ, Clarke BF (1982) Diagnosis and management of diabetic autonomic neuropathy. BMJ 285:916-918
12. Finney DJ (1974) Probit analysis. Cambridge University Press Cambridge UK

13. Luthra A, Borkowski KR, Carruthers SG (1991) Genetic aspects of variability in superficial vein responsiveness to norepinephrine. Clin Pharmacol Ther 49:355-361

14. Ewing DJ, Bellavere F, Epsi F et al. (1986) Correlation of cardiovascular and neuroendocrine tests of autonomic function in diabetes. Metabolism 35:349-353

15. Moorhouse JA, Carter SA, Doupe J (1966) Vascular responses in diabetic peripheral neuropathy. BMJ 1:883-888

16. Neubauer B, Christensen NJ (1976) Norepinephrine, epinephrine and dopamine contents of the cardiovascular system in longterm diabetics. Diabetes 25:6-10

17. Eichler HG, Blaschke TF, Kraemer FB, Ford GA, BlochlDaum B, Hoffman BB (1992) Responsiveness of superficial hand veins to $\alpha$-adrenoceptor agonists in insulin-dependent diabetic patients. Clin Sci 82:163-168

18. Ahmed ME, Le Quesne PM (1986) Quantitative sweat test in diabetics with neuropathic foot lesions. J Neurol Neurosurg Psychiatry 49:1059-1062

19. Deanfield JE, Daggett PR, Harrison MJG (1980) The role of autonomic neuropathy in diabetic foot ulceration. $J$ Neurol Sci 47:203-210

20. Rhie FH, Christlieb AR, Sandor Tet al. (1982) Retinal vascular reactivity to norepinephrine and angiotensin II in normals and diabetics. Diabetes 31:1056-1060

21. Christlieb AR, Janka HU, Kraus B et al. (1976) Vascular reactivity to angiotensin $\Pi$ and norepinephrine in diabetic subjects. Diabetes 25:268-274

22. Tolins PJ, Raij L (1988) Genetic factors and susceptibility to diabetic nephropathy. N Eng J Med 319:180-181 